\title{
Performance of the Horizon-10T detector system in Physics Run 1
}

\author{
Rashid Beisembaev ${ }^{1}$, Dmitriy Beznosko², Kanat Baigarin ${ }^{3}$, Ayan Batyrkhanov ${ }^{3}$, Elena Beisembaeva ${ }^{1}$, Oleg \\ Dalkarov $^{1}$, Alexander lakovlev ${ }^{2, \star}$, Vladimi Ryabov ${ }^{1}$, Turlan Sadykov ${ }^{4}$, Sergei Shaulov ${ }^{1}$, Marina Vildanova ${ }^{1}$, Tileubek \\ Uakhitov $^{3}$, and Valeriy Zhukov ${ }^{1}$ \\ ${ }^{1}$ P. N. Lebedev Physical Institute of the Russian Academy of Sciences, Moscow, Russia \\ ${ }^{2}$ Bard Early College New Orleans, New Orleans, USA \\ ${ }^{3}$ Nazarbayev University, Astana, $K Z$ \\ ${ }^{4}$ LLP "Institute of Physics and Technology", Almaty, Kazakhstan
}

\begin{abstract}
.
This article overviews the performance of the Horizon-10T (H10T) detector system in Physics Run 1. The upgraded H10T detector system consists of ten charged particle detection points, separated by distances from a hundred meters up to 1.3 kilometer and is able to detect disks in primary particle energy ranges of $10^{16}-10^{19} \mathrm{eV}$ and zenith angles from $0^{\circ}$ to $85^{\circ}$ with a few ns precision.
\end{abstract}

\section{Introduction}

Horizon-10T (H10T) [1] detector system is an upgrade from the original Horizon-T (HT) [2,3] experiment and is designed to further study the longitudinal and latitudinal structure of charged particles in Extensive Air Shower (EAS) disks. It is located at the territory of the Tien Shan high-altitude Science Station at 3340 meters above sea level. This station is part of Lebedev Physical Institute of the Russian Academy of Sciences. During the upgrade, two new detection points have been installed. Now H10T consists of ten charged particle detection points that all have scintillator-based detectors and some have glassbased ones. The Horizon-10T detector system aerial view is presented in Figure 1.

\section{Detector System Description}

The H10T detector system consists of detectors of several different types: plastic scintillator with Hamamatsu R7723 PMT detectors and glass with R7723 PMT detectors in detection points 1 through 8 , plastic scintillator with Hamamatsu H6527 PMT in detection points 9 and 10, and a Vavilov-Cherenkov radiation detector with H6527 PMT assemblies that is located in detection point 1 (center).

The H10T detector system is based on detectors of two different types: all detection points have one scintillator detector (SD) parallel to the sky. Each SD uses polystyrene-based square-shaped cast scintillator [4] with $1 \mathrm{~m}^{2}$ area and $5 \mathrm{~cm}$ thickness and 2-inch Hamamatsu [5] R7723 photoelectrical multipliers (PMT) register scintillator light except for points 9 and 10 that use H6527 PMT.

\footnotetext{
^e-mail: alexander.iakovlev@ dozory.us
}

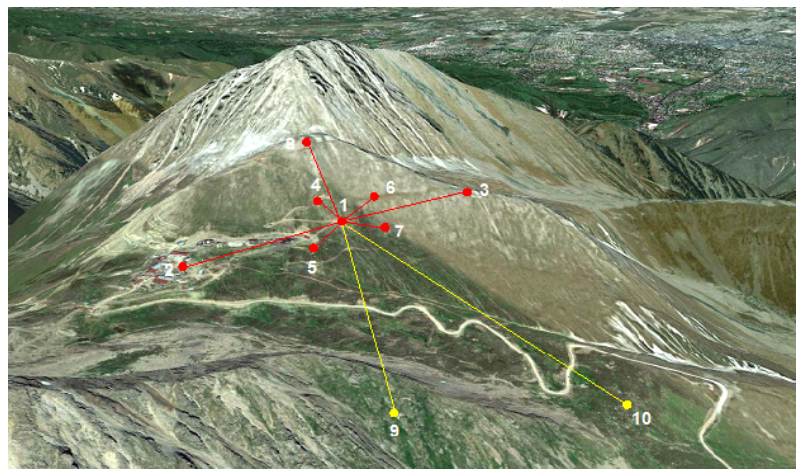

Figure 1. Horizon-10T detector system. A view from above.

Detection points 1-3 have PMT-49 (FEU49) from MELZ [6] (a $15 \mathrm{~cm}$ diameter spherical-shaped cathode PMT with a spectral response from $360 \mathrm{~nm}$ to $600 \mathrm{~nm}$ ) installed alongside the R7723 PMT so cross-calibration between these PMTs is possible. Detection points 9 and 10 use a SD with $12.7 \mathrm{~cm}$ Hamamatsu H6527 PMTs only. Additionally, points 1 and 4-7 have fast time resolution glass-based detectors (GD) [7] with R7723 PMT for readout. A typical SD is shown in the Figure 2.

H10T also has the Vavilov-Cherenkov radiation detector sub-system (VCD) (Figure 3) which is located next to detection point 1 . It is used for EAS Cherenkov light registration with a comparable time resolution of a few ns. Note that the optical detector was not used in physics run 1. VCD consists of three parabolic mirrors of $150 \mathrm{~cm} \mathrm{di-}$ ameter and focal length of $65 \mathrm{~cm}$. They are mounted on the rotating support allowing detection in the zenith angle range of $0^{\circ}-80^{\circ}$ and in azimuthal angle range of $0^{\circ}-360^{\circ}$. 


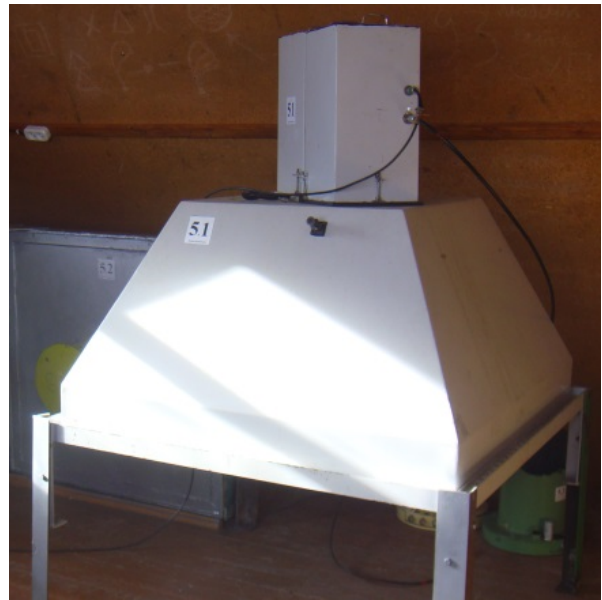

Figure 2. A typical SD detector

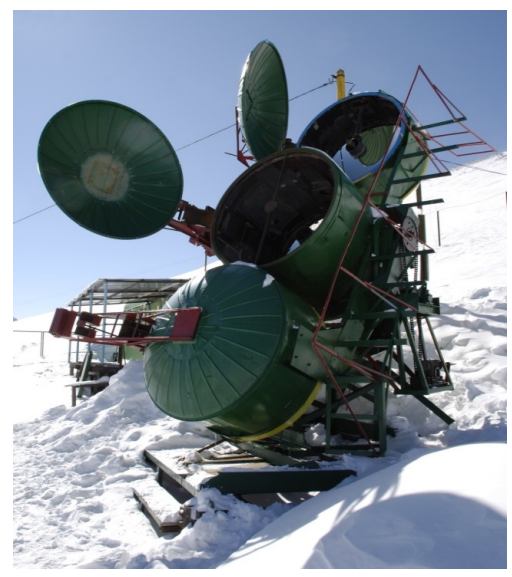

Figure 3. VCD sub-system

A H6527 PMT is located in the focal point of each mirror. The field of view of each mirror + PMT is $\sim 13^{\circ}$.

\section{Data acquisition}

All PMT signals are carried over the coaxial cables RK 75-7-316F-C SUPER produced by SpetsKabel [8] and impedance matched to the rest of the electronics and calibrated [9]. Three 14-bit CAEN [10] DT5730 ADC (analog to digital converter) boards in a common trigger scheme comprise the data acquisition system (DAQ) that is located at detection point \#1. The system-wide electronic trigger is formed by a first ADC board. The waveform recorded by each ADC channel consist of 5110 bins digitized every $2 \mathrm{~ns}$ each, for a total of $10.22 \mu \mathrm{s}$. The larger time ranges are possible with up to 2 million data points per channel per event.

The DAQ is triggered when detection points 4 and 7 report the passage of more than $\sim 5$ charged particles. This liberal initial hardware trigger allows keeping a larger data sample for further offline analysis. A typical offline trigger requires a signal from all four detection points: $4,5,6 \& 7)$. All detectors are calibrated to single MIP re-
Table 1. H10T shower detection threshold energy dependence at different zenith angles

\begin{tabular}{ll}
\hline Zenith angle & Threshold $\mathrm{E}_{\mathrm{o}}, \mathrm{eV}$ \\
\hline $0^{\mathrm{o}}-30^{\circ}$ & $10^{16}-2 \cdot 10^{16}$ \\
$45^{\circ}$ & $5 \cdot 10^{16}$ \\
$60^{\circ}$ & $10^{17}$ \\
$70^{\circ}$ & $10^{18}$ \\
$80^{\circ}$ & $2 \cdot 10^{18}$ \\
\hline
\end{tabular}

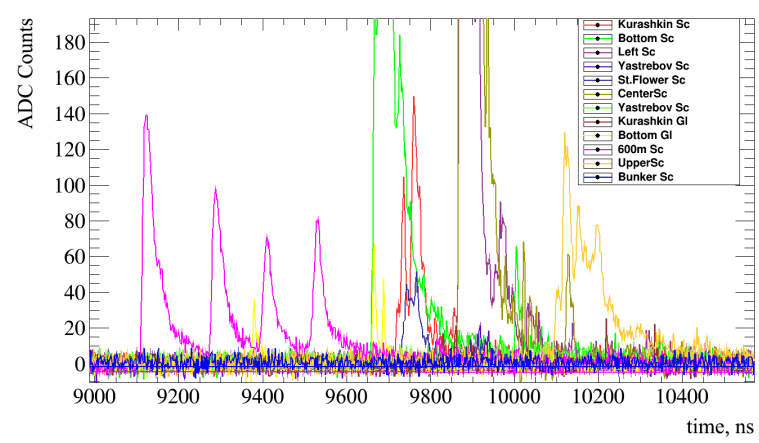

Figure 4. Example of UE events in the H10T data display

sponse that also sets pulse timings and connection cables properties [1].

\section{Detection Energy Threshold and Area Acceptance}

The H10T shower detection threshold energy dependence at different angles has been obtained using the CORSIKA [11] simulation package and is provided in Table 1.

The threshold grows slowly between $0^{\circ}$ and $30^{\circ}$, and then goes up about 50 times between $30^{\circ}$ and $70^{\circ}$. Above $70^{\circ}$, the threshold growth is slowed since around that angle value the muon fraction of the EAS disk is approaching the electron one and then prevailing.

The geometric factor $\Gamma\left(\mathrm{E}_{\mathrm{o}}\right)$ of the H10T detector system for EAS detection at different primary energies is provided in Table 2 . With increasing zenith angles, both the detection threshold energy and the collection area grow. Therefore, as $\mathrm{E}_{\mathrm{o}}$ increases, both the area $\mathrm{S}$ and the detector system registration solid angle $\Omega$ grow as well. Table 2 also lists the event rate $\mathrm{N} / \mathrm{t}$ of all events above $\mathrm{E}_{\mathrm{o}}$.

\section{EAS events detected}

The previous HT results about the Unusual Events (UE) has been published in $[12,13]$. The name 'unusual' is used in place of previously used common names such as 'delayed particle' or 'multimodal' events.

The H10T upgrade has been carried out to extend the capability for UE detection. An example of the H10T data displays of an 'unusual' event is presented in Figure 4.

Multi-modal signals in several channels can be seen. The separation increases with distance from the EAS core. $\mathrm{X}$-axis is the time in $\mathrm{ns}, \mathrm{y}$-axis is ADC bins. 
Table 2. The geometric factor $\Gamma\left(\mathrm{E}_{\mathrm{o}}\right)$ of the $\mathrm{H} 10 \mathrm{~T}$ detector system for EAS detection at different primary energies

\begin{tabular}{lllllll}
\hline $\mathrm{E}_{\mathrm{o}}, \mathrm{eV}$ & $2 \cdot 10^{16}$ & $5 \cdot 10^{16}$ & $10^{17}$ & $2 \cdot 10^{17}$ & $10^{18}$ & $10^{19}$ \\
\hline$\Gamma\left(\mathrm{E}_{\mathrm{o}}\right), \mathrm{km}^{2} \mathrm{sr}$ & 0.45 & 0.64 & 1.00 & 1.45 & 3.65 & 11.69 \\
$\frac{N}{t}\left(>E_{o}\right), \frac{\text { event }}{\text { hour }}$ & 8.6 & 1.49 & 0.54 & 0.18 & 0.016 & $4 \cdot 10^{-4}$ \\
\hline
\end{tabular}

For physics run 1 (Nov 2017- May 2018) the total detector uptime was $\sim 4200$ hours with a total of $\sim 25000$ events detected. About 8000 events are selected by the offline trigger with about a percent of them being UE.

\section{Conclusion}

Physics run 1 has been completed successfully on the H10T - an upgrade from HT detector system. Unusual EAS events are detected with pulses clearly showing several maxima.

The ten detection points of H10T have charge particle detectors separated by hundreds of meters that allow determination of temporary characteristics of EAS with timing precision of $\sim 2 \mathrm{~ns}$. Pulses from each detector are digitized with $2 \mathrm{~ns}$ steps for a total of 5110 bins per event. The ADCs have 14-bit dynamic range.

The H10T physics run 1 lasted $>4000$ hours and yielded about 8000 events. From this data, up to a hundred multi-modal events have been found with pulse modes separated by hundreds of ns.

\section{References}

[1] D Beznosko et al., arXiv:1803.08309 (2018)
[2] R U Beisembaev et al., arXiv:1605.05179 (2016)

[3] D Beznosko et al., EPJ Web of Conferences 145, 11004 (2017)

[4] A Baitenov et al., arXiv:1601.00086 (2016)

[5] Hamamatsu Photonics, 314-5 Shimokanzo, Toyookavillage, Iwatagun, Shizuoka-ken, 438-0193 Japan http://www.hamamatsu.com

[6] MELZ-FEU, 4922-y pr-d, 4c5, Zelenograd, Moscow, Russia, 124482 http://www.melz-feu.ru

[7] R U Beisembaev, et al., Journal of Instrumentation 12, no. T07008 (2017)

[8] SpetsKabel Inc., 6/1-5 Birusinka St., Moscow, Russia http://www.spetskabel.ru

[9] T Beremkulov et al., arXiv:1608.04312 (2016)

[10] CAEN S.p.A. Via della Vetraia, 11, 55049 Viareggio Lucca, Italy http://www.caen.it

[11] D Heck et al., Forschungszentrum Karlsruhe Report FZKA 6019 (1998)

[12] D Beznosko et al., EPJ Web of Conferences 145, 14001 (2017)

[13] R U Beisembaev et al., accepted to Journal of Physics: Conference series (2018) 